\title{
NOVEL EVALUATIONS OF PROTOCATECHUIC ACID TO TARGET IL-17A FOR PSORIASIS PROTECTION IN MICE
}

\author{
JIRU DU ${ }^{1 *}$, MIAO QIAN ${ }^{1 *}$, YUAN TING ZHI ${ }^{1 *}$, WEI LIU ${ }^{1}$ and NINGJING SONG ${ }^{1 *}$ \\ ${ }^{1}$ Department of Dermatology, Tongren Hospital, \\ Shanghai Jiao Tong University School of Medicine, Shanghai, 200336, China
}

\begin{abstract}
Psoriasis is an erythrogenic skin condition identified by multi-layered scales in addition to the thick epidermis (top layer of the skin). The function of interleukin-17A (IL-17A) in the progression of psoriasis is well-known. The interactivity of IL-17A with IL-17(R)A is the main target to investigate the interaction between the two interleukins, which resulted in the amelioration of psoriasis in mice. Our study was aimed to analyze the PCA effects on the association between IL-17A and IL-17(R)A in psoriasis induced mouse model by imiquimod (IMQ). The topical use of IMQ caused psoriasis in mice, followed by oral administration of two doses of PCA $(-50$ and $-100 \mathrm{mg} / \mathrm{kg})$ respectively for fourteen successive days. IL-17A blocking assay was performed to analyze the relationship between IL-17A and IL-17(R)A, followed by RNA- sequencing and histopathological studies. The blocking assay of IL-17A demonstrated that PCA was able to interrelate with the IL-17A residues and therefore eliminated the binding capacity of IL-17(R)A to IL-17A. PCA, in a dose-dependent manner, changed the interrelationship between IL-17A and IL-17(R)A; diminished IMQ-caused mice's skin lesions, and also improved histopathological lesions. PCA diminished the invasion of the inflammatory cells and cytokine secretions in IMQ- treated psoriasis. Our results identified novel findings involving oral administration of protocatechuic acid as a bioactive compound that offered protection against IMQ-caused psoriasis in mice through the alteration of interaction between interleukin-17A and IL-17(R)A.
\end{abstract}

Keywords: Protocatechuic acid, interleukin-17A, skin inflammation, imiquimod, psoriasis

Protocatechuic acid (PCA) is a phytochemical abundant phenolic acid, generally present in plants, vegetables, and fruits $(1,2)$. PCA has diverse pharmacological actions such as anti-oxidant, anti-inflammatory, anti-microbial, and neuroprotective function $(1,2)$. PCA shows antioxidant effects and mitigates redox stress induced by doxorubicin and oxidative stress in myocardial cells (3). PCA provides protection against splenotoxicity caused by hyperglycemia and oxidative stress $(4,5)$. PCA exhibits neuroprotective activity in rats following cerebral ischemia/reperfusion damage against oxidative stress-mediated apoptosis (6). PCA prevents dopaminergic neurodegeneration caused by antioxidant, anti-inflammatory activity by rotenone, suggesting that PCA can be used as an effective neuroprotective agent to prevent Parkinson's disease (7). PCA also plays a crucial role in several pathophysiological disorders as an antiinflammatory agent including hepatic and aortic inflammation, ulcerative colitis, and ultraviolet-caused skin inflammation $(8,2,9)$.
Psoriasis is a common skin condition affecting approximately 2 percent of the population worldwide. This is distinguished by highly defined, eczematous, plagues of scales that usually damage the scalps, elbows, and so on. People with this condition are also likely to develop rheumatoid arthritis, heart problems, and metabolic disease (10). The psoriasis etiology is rather complex but recent work has shown that interleukin-17A acts a significant role in the progression of psoriasis (11). The psoriasisrelated gene expression in keratinocytes is caused by interleukin-17A or otherwise along with the tumor necrosis factor- $\alpha$. Interleukin-17A also activates signaling molecules such as NF- $\kappa \mathrm{B}$, contributing to proliferation and irregular differentiation of keratinocytes and invasion of lymphocytes (11). Psoriasis treatment centers used topical applications such as corticosteroids, analogs of vitamin D, coal tars, etc., photodynamic therapy, and clinical medicine (such as methotrexate, immunosuppressive medications and biological) (12). Although such medications have

\footnotetext{
* Corresponding author: snj3367@shtrhospital.com

\# These authors contributed to this work equally
} 
positive benefits on psoriasis, they also have unintended harmful effects. Conversely, immunotherapy including interleukin-17A may induce inflammation of the upper respiratory and lower alimentary tracts and intermittent neutropenia $(13,14)$.

We also observed in previous studies that vanillin inhibits psoriatic inflammatory response in mice, is related to regulation IL-17 (15). The latest study suggested that PCA prevents the expression of IL17A mediated synoviocytes obtained from arthritis (15). PCA's effects on interaction with interleukin17A / interleukin-17(R)A and its related disorders, such as psoriasis, remain unclear. We assessed the effects of PCA on the interleukin-17A and interleukin17(R)A relationship by an interleukin-17A-blocking assay to attend to this question. The advantageous impact of PCA on psoriasis was analyzed in imiquimod-treated psoriasis. Assessment of histopathological lesions in inflammation similar to psoriasis indicated improvement with PCA. PCA's effect on psoriasis was further explored by the method of RNA-sequencing and IHC (immunohistochemical) staining. The study results demonstrated that PCA significantly strengthened the association between interleukin-17A and interleukin-17(R)A, leading to an improvement in psoriasis-like inflammation in mice.

\section{EXPERIMENTAL}

\section{Chemicals}

Imiquimod (IMQ, 5\% cream) was purchased from Qingdao Franken International Co., Ltd. Shandong, China. Protocatechuic acid (PCA) was purchased from Fine Chemicals, Ltd, China. Petroleum Jelly (Vaseline) was procured from the local market. Horseradish peroxidase (HRP), TMB (3, 3', 5, 5'- Tetramethylbenzidine), goat anti-human IgG-Fc was obtained from Thermofisher Scientific, China. Mouse monoclonal antibody against CD11c and polyclonal antibodies (rabbit) against IL-17A and IL-1 $\beta$ were obtained from Santa Cruz Biotechnology (Shanghai) Co., Ltd. Monoclonal and polyclonal antibodies of rabbit against IL-23, CD4, and CD11b and monoclonal antibodies of rat against Gr-1 were purchased from Abcam Ltd, China along with, recombinant proteins such as IL-17A and IL-17RA (Fc active).

\section{Blocking assay of interleukin-17A}

At $4{ }^{\circ} \mathrm{C}$, micro-titer plates were coated with one nanogram per well containing recombinant interleukin-17A dissolved in PBS (Phosphate buffer saline, $\mathrm{pH}$ 7.4). Plates were rinsed thoroughly for four times with $500 \mu \mathrm{L}$ per well of phosphate buffer saline containing $1 \%$ of Tween 20 , blocked with $200 \mu \mathrm{L}$ per well of phosphate buffer saline containing 1\% Tween 20 and $2 \%$ bovine serum albumin acting as a blocking buffer at $37^{\circ} \mathrm{C}$ for $45 \mathrm{~min}$, followed by incubation with several a positive control (IL-17R) or PCA concentrations at $37^{\circ} \mathrm{C}$ for $2 \mathrm{~h}$. The recombinant Fc-tag interleukin-17(R)A, which was diluted by blocking buffer, was incorporated followed by incubation at room temperature for two hours after washing with phosphate buffer saline containing 1\% Tween 20 for four times. The bound Fc-tag interleukin-17(R)A was again determined by the addition of $200 \mu \mathrm{L}$ per well containing HRP-conjugated goat anti-human IgG Fc antibody at $37^{\circ} \mathrm{C}$ for $2 \mathrm{~h}$ and $200 \mu \mathrm{L}$ per well of TMB single solution for $30 \mathrm{~min}$. The reaction was terminated by the addition of $50 \mu \mathrm{L}$ of sulphuric acid $(2 \mathrm{~N})$ per well in an ELISA plate reader and the absorbance was recorded at $420 \mathrm{~nm}$. Interleukin-17A blocking capability was demonstrated by modifications in optical density (OD) determined by deducting absorbance without PCA or interleukin-17 (R) A from the absorbance at the specified PCA or interleukin-17 (R) A concentration.

\section{Animal experimentation}

Animal Research Center (Shanghai, China) contributed BALB/c mice (8 weeks old, either sex). Mouse experiments were performed after obtaining permission from the Ethics Committee from the Institutional Committee on Animal Welfare. Mice were preserved under a light / dark period of 12 : $12 \mathrm{~h}$, with free exposure to food and water. They were classified arbitrarily into four groups of eight mice per group.

(1) Control treated with petroleum jelly (Vaseline)

(2) IMQ-induced psoriatic mouse model

(3) IMQ-induced psoriasis $+50 \mathrm{mg} / \mathrm{kg}$ PCA treatment

(4) IMQ-induced psoriasis $+100 \mathrm{mg} / \mathrm{kg}$ PCA treatment.

In mice, inflammation of the skin similar to psoriasis was caused as previously described in the study $(16,17)$. In brief, with the aid of Veet $\AA$, the hair on the dorsal side was trimmed off. Vaseline (Control) or imiquimod creams (Psoriatic mouse model) were dispersed topically on the dorsal side of the mouse's skin over an area of $3 \times 2$ sq. cm for 14 days. PCA (50, or $100 \mathrm{mg} / \mathrm{kg}$ ) was given regularly for over 2 weeks. Depending on the area of psoriasis and the severity index (PASI), the degree of inflammation on the dorsal skin was graded every two days. Blind and scored erythema (redness) and desquamation (scaling) separately on a scale ranging from zero to four: zero indicates no psoriasis; one 
indicates slightly developed psoriatic inflammation; two indicates moderately developed psoriatic inflammation; three indicates markedly developed psoriatic inflammation and four indicates severely developed psoriatic inflammation. On the fourteenth day, mice were sacrificed. Skin specimens were taken for further assessments.

\section{Histopathological evaluation}

The skin tissues were placed in $10 \%$ formalin, paraffin-fixed, cut into $5 \mu \mathrm{m}$ thickness sections, and treated with hematoxylin/eosin stain. Two experts examined blindly the histopathological modifications. Image Scope (Olympus, China) was used to measure the thickness of the epidermis for approximately 30 40 random sample measurements.

\section{RNA sequencing}

An RNeasy Mini Kit (Cell Signaling Engineering, China) was used to isolate total RNA from $40 \mathrm{mg}$ of skin tissue was tested using a bio-analyzer for RNA concentration and quality. In short, total RNA was processed with magnetic oligo (dT) beads to choose the mRNA. Random N6 primers were used to reverse the transcription of mRNA fragments into double-stranded cDNA. The synthesized cDNA was end-repaired, with 3 ' adenylation. Adapters were attached to the ends of such $3^{\prime}$ adenylated cDNA components, and the polymerase chain (PCR) reaction amplified the ligation products to enhance the refined cDNA templates. The PCR elements were then heat-denatured, and the oligo-chain splint circularized. Bioanalyser was used to validate the fragment length. For this experiment, we used three bio-replicates.

\section{Immunohistochemical staining}

Sequentially, the skin tissue sections of approximately 6-7 $\mu \mathrm{m}$ thickness were incubated with primary antibodies overnight at a temperature of $4^{\circ} \mathrm{C}$ after dilution to 1: 300 followed by secondary antibodies biotinylated and complex reagent avidin-biotin were incubated at $37^{\circ} \mathrm{C}$ for 10 minutes respectively. These sections were stained with 3, 3'-diaminobenzidine and examined using ImageJ for positive IL-(17A, -23 and $-1 \beta$ ) regions. Positive areas were determined by the brown color area occupied to the total tissue area multiplied by 100 . The positive cell (CD11b, CD11c, CD4, and Gr-1) proportions were measured by counting the brown cells occupied in the total tissue area multiplied by 100 . A minimum of hundred cells was counted for every observation.

\section{Statistical analysis}

Data was described as a mean \pm standard error. Results were analyzed using SPSS Statistics, version 20 with one-way variance analysis (ANOVA) and posthoc Bonferroni analysis. A p-value $<0.05$ was found statistically significant.

\section{RESULTS}

\section{PCA modified the relationship of interleukin- 17A with interleukin-17(R)A}

Interleukin-17A acts an incredibly significant role in the initiation and progression of psoriasis. We examined if PCA interfered with interleukin-17A and inhibited interleukin-17A coupling to interleukin17(R)A. Therefore interleukin-17A blocking assay was performed. An ELISA sandwich was used to identify the compound that can inhibit interleukin17A interaction with interleukin-17(R)A. As illustrated in Figure 1, interleukin-17(R)A (positive control) attached to interleukin-17A and thus removed the

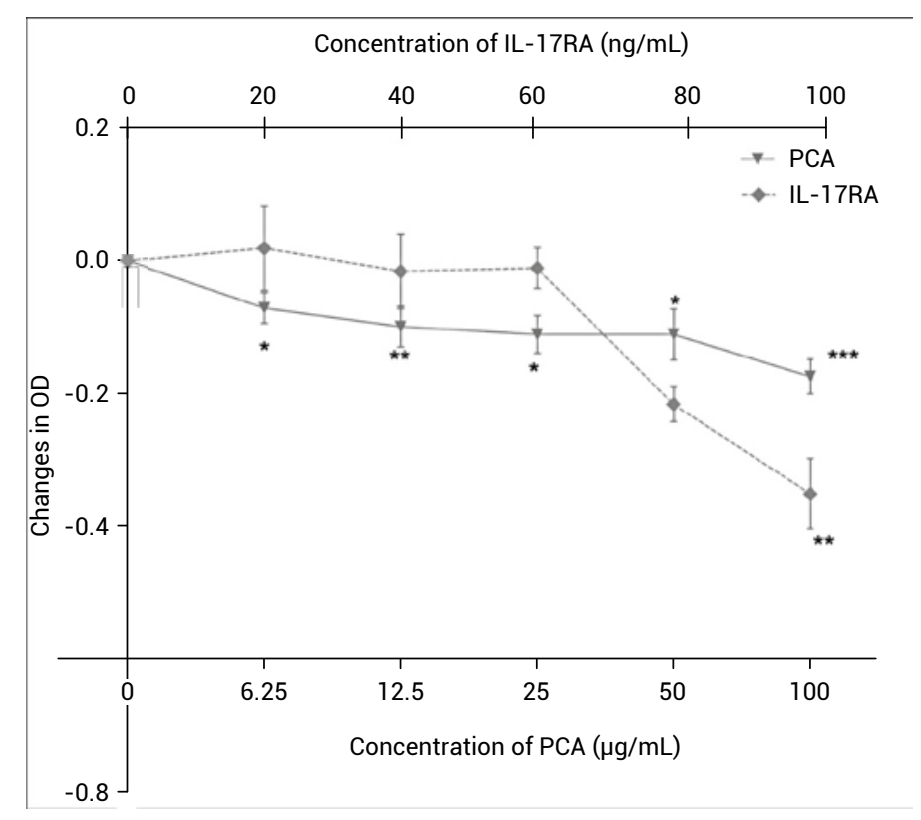

Figure 1. IL-17A-blocking assay demonstrating possible PCA and IL-17A interaction. Specific concentrations of IL-17RA and PCA were added to the wells coated with IL-17A. After incubating at room temperature for $1 \mathrm{~h}$, sequentially added Fc-tag IL-17RA, HRP-conjugated anti-human IgG Fc goat antibody, and chromatic substrate. The absorbance was recorded at $420 \mathrm{~nm}$. IL-17A-blocking capability was determined by deducting absorbance at the specified concentration of IL-17RA or FA from the absorbance without IL-17RA or PCA. Values of three independent assays are represented as mean \pm standard error. ${ }^{*} \mathrm{p}<0.05$ and $* * \mathrm{p}<0.01$, compared to without IL-17RA or PCA. 

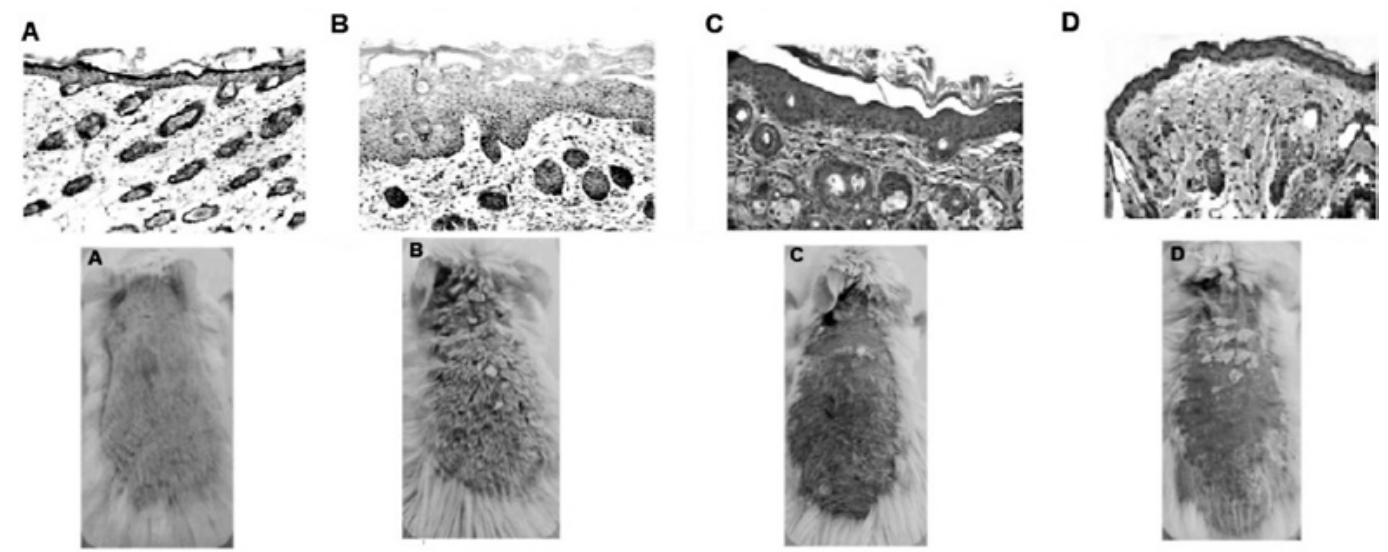

Figure 2. Effect of protocatechic acid in A) control mice showing no histopathological changes. Mice were applied topically to dorsal skin using Vaseline petroleum Jelly (control). B) IMQ induced psoriasis showing histopathologic lesions in mice. Mice were topically applied to dorsal skin using IMQ cream (Model). C) Treatment group showing remarkable improvement in the histopathological lesions in mice caused by IMQ, by treatment with PCA $-50 \mathrm{mg} / \mathrm{kg}$ administered to mice orally for 14 consecutive days. D) Treatment groups showing significant improvement in the histopathologic lesions in mice caused by IMQ, by treatment with PCA-100 mg/kg administered to mice orally for 14 consecutive days. H\&E staining of dorsal skin tissue (original magnification of $200 \mathrm{x}$ ). Photographs are representative histopathological images ( $\mathrm{n}=8 \mathrm{mice} /$ group). Bar Scale $=100 \mu \mathrm{m}$.

Fc-tag interleukin-17(R)A adhesion to interleukin$17 \mathrm{~A}$ ensuing in a momentous reduction in optical density values. The effect of PCA often considerably reduced OD levels, and a dose-dependent manner accompanied the decline. Such results showed that PCA might interfere with interleukin-17A, and modify the interleukin-17A / interleukin-17(R)A relationship.

\section{PCA mitigated skin lesions similar to psoriasis induced by IMQ}

Psoriasis is a common inflammatory skin condition, primarily caused by interleukin-17A. We were allowed to treat mice with IMQ topical application on their dorsal skin and administered two doses of protocatechuic acid (50 and $100 \mathrm{mg} / \mathrm{kg}$ ) to mice orally for fourteen days to explore the impact of PCA on IMQ-treated psoriasis. The extent of inflammation in the skin was measured by PASI on each second day. As illustrated in Figure 2a; Table 1 and 2, smooth dorsal skin appearance of vaseline-treated mice (control group) and no signs of scaling or redness were found during the 14-day test. In comparison, dry skin lesions in the dorsal region were seen in animals treated with IMQ (psoriatic mouse model, Figure 2b; Table 1 and 2). From day 2, the signs of scaling and redness were started to notice, as well as the severe symptoms that emerged on day 4. PCA treatment decreased morphological changes caused by IMQ on mice's dorsal skin from day 4 onwards. Upon PCA intervention, lesser scales and reddening were noticed on the dorsal side of the skin (Figure 2c and d; Table 1 and 2). Furthermore, intervention with PCA (50 and $100 \mathrm{mg} / \mathrm{kg}$ ) substantially improved skin lesions from day 8 .

\section{PCA strengthened IMQ-treated psoriasis-like lesions in mice}

Skin tissue portions treated with H\&E were evaluated for histopathologic lesions. As seen in Figure 3, 14-day IMQ intervention, caused

Table 1. Effects of PCA on Erythrema score in groups of mice monitored for 14 days.

\begin{tabular}{|c|c|c|c|}
\hline & Model & PCA-50 mg/kg & PCA-100 mg/kg \\
\hline Day 2 & $1.18 \pm 0.38$ & $2.38 \pm 0.39$ & $1.63 \pm 0.62$ \\
\hline Day 4 & $4.04 \pm 0.62$ & $3.57 \pm 0.49$ & $2.92 \pm 0.57^{*}$ \\
\hline Day 6 & $4.03 \pm 0.52$ & $3.13 \pm 0.43^{*}$ & $2.69 \pm 0.62^{*}$ \\
\hline Day 8 & $4.53 \pm 0.69$ & $3.33 \pm 0.60^{*}$ & $3.25 \pm 0.53^{*}$ \\
\hline Day 10 & $4.02 \pm 0.48$ & $2.14 \pm 0.63^{*}$ & $1.90 \pm 0.46^{*}$ \\
\hline Day 12 & $3.98 \pm 0.48$ & $1.75 \pm 0.66^{*}$ & $1.37 \pm 0.70^{*}$ \\
\hline Day 14 & $2.86 \pm 0.55$ & $0.94 \pm 0.46^{*}$ & $0.75 \pm 0.49^{*}$ \\
\hline
\end{tabular}

Values are mean $\pm \operatorname{SEM}(n=8)$. Statistical significance expressed as ${ }^{*} \mathrm{p}<0.05$ versus sham group compared to PCA-50 $\mathrm{mg} / \mathrm{kg}$ and PCA-100 $\mathrm{mg} / \mathrm{kg}$ groups respectively. 
Table 2. Effects of PCA on Scaling score in groups of mice monitored for 14 days.

\begin{tabular}{|c|c|c|c|}
\hline & Model & PCA-50 mg $/ \mathrm{kg}$ & PCA-100 mg/kg \\
\hline Day 2 & $1.22 \pm 0.53$ & $1.81 \pm 0.34$ & $1.73 \pm 0.72$ \\
\hline Day 4 & $4.83 \pm 0.50$ & $2.97 \pm 0.40^{*}$ & $2.76 \pm 0.53^{*}$ \\
\hline Day 6 & $4.37 \pm 0.80$ & $3.47 \pm 0.30^{*}$ & $3.28 \pm 0.37^{*}$ \\
\hline Day 8 & $4.93 \pm 0.79$ & $2.49 \pm 0.51^{*}$ & $2.29 \pm 0.70^{*}$ \\
\hline Day 10 & $3.68 \pm 0.65$ & $1.72 \pm 0.35^{*}$ & $1.67 \pm 0.54^{*}$ \\
\hline Day 12 & $3.59 \pm 0.62$ & $1.46 \pm 0.56^{*}$ & $1.33 \pm 0.48^{*}$ \\
\hline Day 14 & $2.05 \pm 0.34$ & $0.97 \pm 0.26^{*}$ & $0.94 \pm 0.22^{*}$ \\
\hline
\end{tabular}

Values are mean \pm SEM $(n=)$. Statistical significance expressed as ${ }^{*} \mathrm{p}<0.05$ versus sham group compared to PCA-50 $\mathrm{mg} / \mathrm{kg}$ and PCA-100 $\mathrm{mg} / \mathrm{kg}$ groups respectively.

psoriasis-like lesions including keratosis, subcutaneous dilated capillaries, and inflammatory invasion. The thickness of the epidermis $(54.46 \pm 5.75 \mu \mathrm{m})$ was considerably enhanced relative to control $(6.89 \pm 1.32 \mu \mathrm{m})$ after IMQ treatment (Figure 3). By comparison, oral dosing of PCA (50 and $100 \mathrm{mg} / \mathrm{kg}$ ) continued to improve the IMQ-affected lesions and greatly reduced the thickness of the epidermis formed by IMQ. The epidermis thickness of mice after treatment with PCA $(100 \mathrm{mg} / \mathrm{kg})$ was $32.5 \pm 5.23 \mu \mathrm{m}$, decreased by $67.5 \%$ percent relative to IMQ-induced psoriasis. Such findings revealed that oral dosing of PCA mitigated histopathological alterations in mice's dorsal skin caused by IMQ. Additionally, there was the dose-dependent display of PCA modulation.

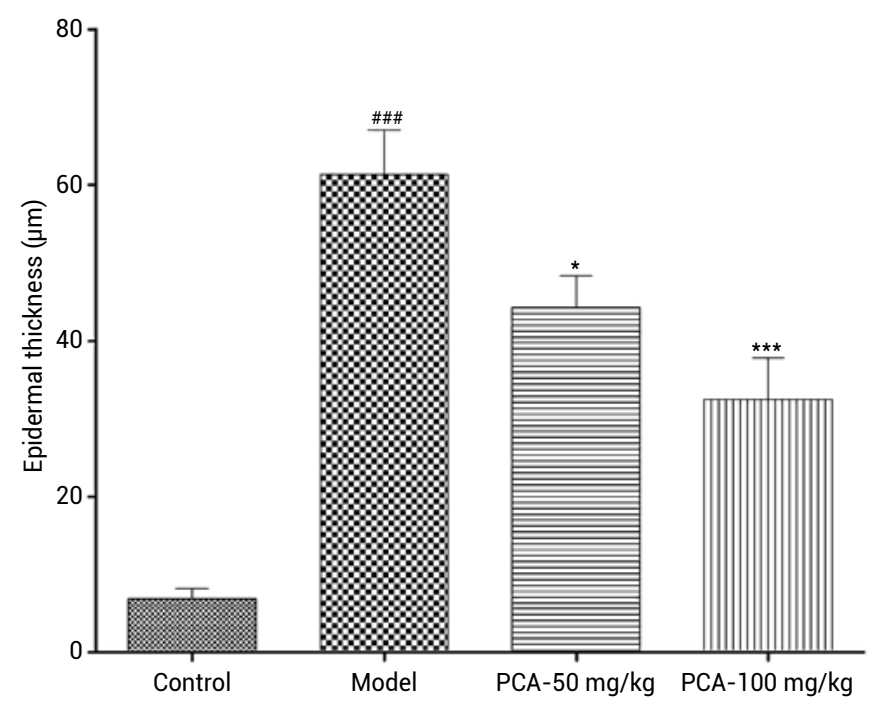

Figure 3. Quantification of epidermal layer thickness. For each group, data were measured by arbitrary measurements of $30-40$. Values are mean \pm standard error. $\# \#$ p $<0.001$, compared to mock group. ${ }^{*} \mathrm{p}<0.05$ and $* * * \mathrm{p}<0.01$, compared to IMQ group.

\section{PCA lowered interleukin gene expression and diminished inflammatory cell infiltration in skin tissue lesions caused by IMQ}

We added IHC staining to further validate the RNA-Sequencing findings. Granulocytes, Leukocytes, dendrites, and $\mathrm{T}$ (helper)-cells were accompanied with the antibodies Gr-1, CD4, CD11b, and CD11c respectively. Helper (T) cells, dendrites expressing IL-23, and granulocytes are identified as contributing factors in pathogenesis for psoriasis. IHC staining was used to monitor invasion and the cytokine secretion of certain cellular subtypes. IMQ enhanced the percentages of CD11c and CD4 positive cells present in the skin surface and gap between both the dermis and the epidermis relative to the control group (Figure 4). The IMQ also enhanced interleukin-17A and IL-23 levels, both in the dermis and in the epidermis. Furthermore, IMQ enhanced the percentages of Gr-1 and CD11b positive cells and the concentrations of IL- $1 \beta$ proinflammatory cytokines predominantly found in the skin surface; unlike IMQ, PCA considerably reduced the percentages of stained cells and interleukin-17A, IL-23, and IL-1 $\beta$ levels in IMQ-caused psoriasis. Such results indicate that, in skin tissues analogous to psoriasis, PCA suppressed the penetration and cytokine release of $\mathrm{T}$ (helper) cells, dendrites, and granulocyte subtypes.

\section{DISCUSSION}

Psoriasis is an inflammation related to skin with a dense epidermis, characterized by multilayered scales. Hitherto, multiple murine-like psoriasis models have been developed including transgenic and xenograft variants. However, owing to the complex pathogenesis of human psoriasis $(18-20)$ 

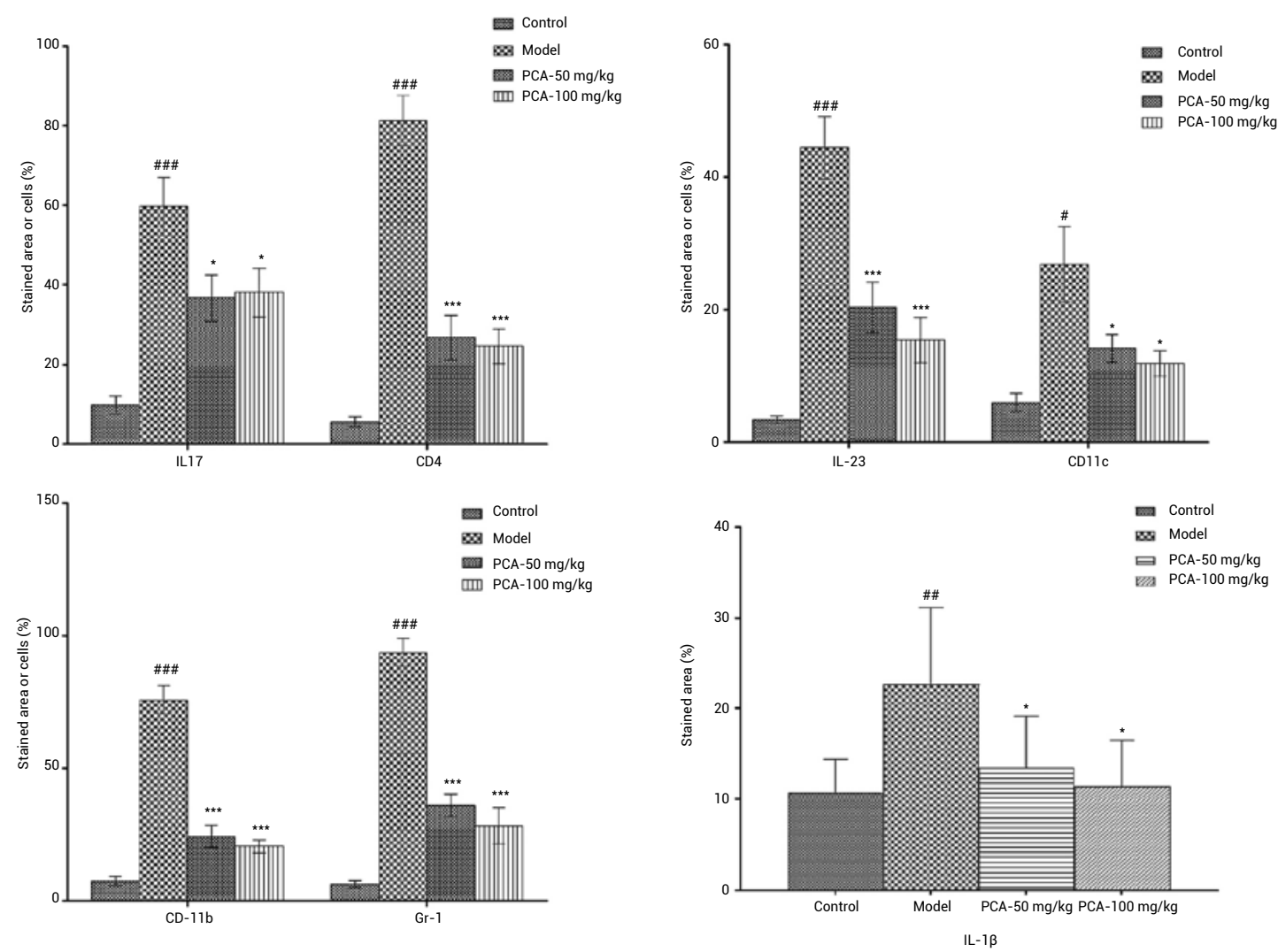

Figure 4. Effect of PCA on the quantification of cytokine secretion of specific antibodies such as IL-17A, CD4, IL-23, CD11c, CD11b, Gr-1, and IL-1 $\beta$. Mice were topically applied with Vaseline (control) or IMQ (Model) on the dorsal skin and/or given orally with PCA -50 and PCA $-100 \mathrm{mg} / \mathrm{kg}$ for 14 consecutive days. Values are mean \pm standard error $(\mathrm{n}=8$ mice per group). \#\#\# $<0.001$, compared to control group. $* \mathrm{p}<0.05 ; * \mathrm{p}<0.01$ and $* * * \mathrm{p}<0.001$, compared to the model group.

these murine-like psoriasis models are confronted with substantial restrictions. For this study, we used the psoriasis model caused by IMQ to determine the impact of PCA on dermatitis which is similar to psoriasis. Despite their reasonable costs, simplicity, ease of use, and fast stimulation of inflammation, IMQ caused intervention has been the commonly employed model for psoriasis. Nevertheless, this paradigm also has certain drawbacks, including the fairly unspecific existence of mediated inflammation of the skin, unfit for therapeutic usage, and improper standard procedures (18-20). Besides, the genetic history of the mice would affect the inflammation similar to IMQ caused psoriasis (21-23, 17). C57BL/6 and BALB/c are two strains of IMQ caused psoriasis used in several studies of mouse lesions. In BALB/c mice, inflammation occurs quicker than in C57BL/6 mice, and adverse effects, like diarrhea and infection, are more chronic in C57BL/6 (17). Nonetheless, modifications in expression in the gene caused by IMQ on the C57BL/6 are significantly correlated with psoriasis than in BALB/c mice (23). We evaluated psoriatic inflammation caused by IMQ in BLAB/c mice in our study since it was simple to view lesions in a lighter color of skin. In fact, along the IL-17 axis, IMQ causes swelling and skin thickening manifestations of psoriasis-like lesions, which resemble the pathogenic mechanisms of human psoriasis.

Because of their antioxidant role, PCA was used as a food substitute (24). It also acts as an anti-inflammatory agent in various tissues, by suppressing cytokine production and reducing oxidative stress. Treatment with PCA defends against acetaminopheninduced damage to the liver through inhibition of Toll-like receptors that mediate inflammatory pathways in mice. PCA increases inflammation of the liver by blocking NF- $\kappa \mathrm{B}$ activation, expression of IL- 6 or TNF- $\alpha$, and the release of free radicals in mice (25). PCA also helps in improving ulcerative colitis by suppressing the production of pro-inflammatory cytokines (IL- 6 , IL- $1 \beta$, TNF- $\alpha$,) and the anti-inflammatory cytokine (IL-10) formation in rats (26). Here we have recently observed that the oral dosing of PCA suppressed inflammation of the psoriatic skin in mice caused by the IMQ. Indeed, IL17 was consistent with its inhibition of inflammation related to the skin. 
IL-17 is a transcription factor that gene was derived from a hybridoma of T-cells of rats and mice (27). To date, there have been six homologous compounds: interleukin-17A, -B, -C, -D, -E, and -F (28). By evaluating RNA-Sequencing, we noticed that the PCA reduced interleukin-17A gene expression, which is the main transcription factor involved in the development and progression of psoriasis. Through a blocking assay with interleukin-17A, we noticed PCA interacting with IL-17 and thus eliminating the Fc-tag with interleukin-17(R)A to interleukin17A. Interleukin-17A binding to interleukin-17(R) A further stimulates the NF- $\mu$ B and STAT cascades which lead to inflammatory gene activation and serial psoriatic disruptions (11). This hypothesis could illustrate how PCA reduced cytokine gene expression, including IL-17 genes, and mitigated mice's skin psoriasis.

Previous research studies suggest PCA has a protective effect on skin disorders. PCA is used to protect the skin against ultraviolet-induced inflammation as a topical antioxidant for hyper-pigmentation and erythema (29). This promotes the healing of wounds in diabetic rats by producing collagen-precursor hydroxyproline and synthesis of hydroxylysine (30). In Swiss albino mice, regulation of inflammatory and apoptotic signals (31) inhibits photocarcinogenesis caused by UV-B radiation. This also inhibits the action of tyrosinase and melanocytic proliferation which results in a reduction in melanogenesis (32). Interestingly, we found in this study, which was consistent with prior work, that PCA had disrupted the pathways involved in melanocyte formation and extracellular receptor interaction. Usually, PCA is used topically to treat skin disease. Our results showed that psoriatic skin inflammation caused by oral administration of PCA increased with IMQ. We suggested that by disrupting interleukin-17A / interleukin-17(R)A interaction, the biodistribution of PCA to spleen after oral administration could influence the population of spleen inflammatory cells and thus increase skin inflammation which is similar to psoriasis.

\section{CONCLUSIONS}

Our findings observed that PCA, in addition to regulating cytokine production, may interfere with interleukin-17A, inhibit interleukin-17A from binding to interleukin-17(R)A, and consequently improve skin inflammation similar to psoriasis. Our results showed that PCA was shown to be a bioactive agent with low toxicity towards psoriatic lesions caused by IMQ at 50 and $100 \mathrm{mg} / \mathrm{kg}$ respectively.

\section{Funding support}

This study was funded by Shanghai Tongren Hospital Project (NO. TRYJ201709).

\section{Acknowledgments}

Not applicable.

\section{Conflicts of interest}

The authors declare no conflict of interest.

\section{REFERENCES}

1. Khan A.K, Rashid R., Fatima N., Mahmood S., Mahmood S., et al.: Acta Pol. Pharm. 72, 643 (2015).

2. Kakkar S., Bais S.: ISRN Pharmacol. 2014, 9 pages (2014).

3. Bhattacharjee N., Dua T.K., Khanra R., Joardar S., Nandy A., et al.: Front. Pharmacol. 8, 251 (2017).

4. Harini R., Pugalendi K.V.: Redox Rep. 15, 71 (2010).

5. Erukainure O.L., Hafizur R.M., Choudhary M.I., Adhikari A., Mesaik A.M., et al.: Biomed. Pharmacother. 86, 307 (2017).

6. Winter A.N., Brenner M.C., Punessen N., Snodgrass M., Byars C., et al.: Oxid. Med. Cell Longev. 2017, 6297080 (2017).

7. Zhang Z., Li G., Szeto S.S.W., Chong C.M., Quan Q., et al.: Free Radic. Biol. Med. 84, 331 (2015).

8. Kore K.J., Bramhakule P.P., Rachhadiya R.M., Shete R.V.: Int. J. Pharm. Life Sci. 2, 909 (2011).

9. Son J.H., Kim S., Jang H.H., Sung N.L., Kyu J.A.: Biomed. Dermatol. 2, 9 (2018).

10. Boehncke W.H., Schon M.P.: Lancet 386, 983 (2015).

11. Hawkes J.E., Yan B.Y., Chan T.C., Krueger J.G.: J. Immunol. 201, 1605 (2018).

12. Conrad C., Gilliet M.: Clin. Rev. Allergy Immunol. 54, 102 (2018).

13. Armstrong A.W., Robertson A.D., Wu J., Schupp C., Lebwohl M.G.: JAMA Dermatol. 149, 1180 (2013).

14. Cui L., Chen R., Subedi S., Yu Q., Gong Y., et al.: Int. Immunopharmacol. 62, 46 (2018).

15. Kelvin A., Nathan R., Arham S., Travis P., Greta V., et al.: Nutrients 12, 1701 (2020).

16. Cheng H.M., Chen F.Y., Li C.C., Lo H.Y., Liao Y.F., et al.: J. Agric. Food Chem. 65, 10233 (2017). 
17. Van D.F.L., Mourits S., Voerman J.S., Kant M., Boon L., et al.: J. Immunol. 182, 5836 (2009).

18. Chuang S.Y., Lin C.H., Sung C.T., Fang J.Y.: Expert. Opin. Drug Discov. 13, 551 (2018).

19. Hawkes J.E., Adalsteinsson J.A., Gudjonsson J.E., Ward N.L.: J. Invest. Dermatol. 138, e1-e8 (2018).

20. Nakajima K., Sano S.: J. Dermatol. 45, 252 (2018).

21. Bezdek S., Hdnah A., Sezin T., Mousavi S., Zillikens D., et al.: Exp. Dermatol. 26, 349 (2017).

22. Flutter B., Nestle F.O.: Eur. J. Immunol. 43, 3138 (2013).

23. Swindell W.R., Michaels K.A., Sutter A.J., Diaconu D., Fritz Y., et al.: Genome Med. 9, 24 (2017).

24. Nankar R., Prabhakar P.K., Doble M.: Phytomedicine 37, 10 (2017).
25. Das U., Manna K., Sinha M., Datta S., Das D.K., et al.: PLoS One 9, e97599 (2014).

26. Sadar S.S., Vyawahare N.S., Bodhankar S.L.: EXCLI J. 15, 482 (2016).

27. Rouvier E., Luciani M.F., Mattei M.G., Denizot F., Golstein P.: J. Immunol. 150, 5445 (1993).

28. Gaffen S.L.: Curr. Opin. Immunol. 23613 (2011).

29. Daréa R.G., Oliveiraa M.M., Truitia M.C.T., Nakamura C.V., Ximenes V.F., Lautenschlager S.O.S.: J. Photochem. Photobiol. B 203, 111771 (2020).

30. Yadav E., Singh D., Yadav P., Verma A.: Biomed. Pharmacother. 96, 86 (2017).

31. Sevgi K., Bektas Tepe B., Sarikurkcu C.: Food Chem. Toxicol. 77, 12 (2015).

32. Zengin G., Senkardes I., Mollica A., Picot-Allain C.M.N., Bulut G., et al.: Comput. Biol. Chem. 75, 111 (2018). 